\title{
ON A FAMILY OF ELLIPTIC SURFACES WITH MORDELL-WEIL RANK 4
}

\author{
CHARLES F. SCHWARTZ
}

(Communicated by William C. Waterhouse)

\begin{abstract}
In this paper, we find bases for the Mordell-Weil groups of a family of elliptic surfaces. In particular, let $E_{(a, b)} \rightarrow B$ be the elliptic surface given by

$$
y^{2}=4\left[x^{3}-\sum_{i=0}^{2} a_{i} u^{i} x+\sum_{j=0}^{3} b_{j} u^{j}\right] .
$$

If the elliptic surface has Mordell-Weil rank 4 over $\mathbf{C}$, then we find a basis $\left\{\sigma_{i}=\left(x_{i}, y_{i}\right) \mid 1 \leq i \leq 4\right\}$ with $x_{i}$ and $y_{i}$ linear in $u$. We do this by finding a parametrization of this family of elliptic surfaces; furthermore, if the parameters are rational numbers, then the Mordell-Weil group is rational over Q.
\end{abstract}

1. Introduction. In 1954, Néron [8] proved that there exist elliptic curves over $\mathbf{Q}$ with rank greater than 10. Since then, Penney and Pomerance [9], Grunewald and Zimmert [3], Brumer and Kramer [1], and Nakata [6] have published examples of curves of rank at least $7,8,9$, and 9 , respectively. We are interested in a corresponding problem for elliptic surfaces (restrict attention to the cases when the $J$-invariant is nonconstant): it can be shown that, if the elliptic surface $E \rightarrow \mathbf{P}_{\mathbf{1}}(\mathbf{C})$ has geometric genus $p_{g}$ equal to zero, then the rank of the Mordell-Weil group (over $\mathbf{C}(u)$ ) is less than or equal to 8. Can one find examples of elliptic surfaces with $p_{g}=0$, and high Mordell-Weil rank over $\mathbf{Q}(u)$ ? Néron [7] proved the existence of surfaces with rank equal to 8 (see also Manin [5]); Brumer has found examples with rank greater than 6; and Schwartz [12], using the method of Néron and Manin, found an example with rank (over $\mathbf{Q}(u)$ ) equal to 8 .

In $\S 2$, we give a parametrized family of elliptic surfaces with Mordell-Weil rank (in general) 4 over $\mathbf{C}(u)$, such that if the parameters are in $\mathbf{Q}$, then all sections are rational over $\mathbf{Q}(u)$. Each member of this family has $p_{g}=0$, and the Néron nonsingular model of each member has a singular fiber of Kodaira type $\mathrm{I}_{0}^{*}$.

In $\S 3$, we find generators for the Mordell-Weil group of every elliptic surface with $J \notin C, p_{g}=0, r=$ Mordell-Weil $\mathrm{rank}=4$, and a singular fiber (in its Néron model) of type $\mathrm{I}_{0}^{*}$. In this section we refer to a result of Schwartz [11] concerning the basis of a generic member of the family of elliptic surfaces, with $J \notin \mathrm{C}, p_{g}=0, r=4$, and a fiber to type $\mathrm{I}_{0}^{*}$.

We will make use of the following theorem, due to Shioda.

Received by the editors September 24, 1984.

1980 Mathematics Subject (Classification (1985 Revision). Primary 14J05, 14J25; Secondary $10 \mathrm{~B} 10$.

Key words and phrases. Elliptic surface, Mordell-Weil group, rational section, rational solution. 

let

THEOREM 1.1. Let $E \rightarrow B$ be an elliptic surface with nonconstant $J$-invariant;

$p_{g}=$ geometric genus of $E$;

$r=$ rank of the Mordell-Weil group;

$\nu_{1}=$ the number of singular fibers of the Néron model of type $\mathrm{I}_{b}, b>0$;

$\nu_{2}=$ the number of singular fibers of the Néron model of other types; and

$g=$ genus of the base, $B$.

Then

$$
r \leq-4+4 g+\nu_{1}+2 \nu_{2}-2 p_{g}
$$

Furthermore, if $p_{g}=0$, then

$$
r=-4+4 g+\nu_{1}+2 \nu_{2}
$$

ProOF. The first statement is Corollary 2.7 of [13]; the second is Remark 3.23 of [2].

One needs a method of classifying the singular fibers of the Néron model. For this, we refer the reader to $[\mathbf{1 0}]$.

Remarks on notation: $\mathbf{Q}, \mathbf{C}, \mathbf{Q}(u)$, and $\mathbf{C}(u)$ will stand for the field of rational numbers, the field of complex numbers, the field of rational functions in one variable over the rational numbers, and the field of rational functions over the complex numbers. We will use the "section at $\infty$ " as the zero section of the Mordell-Weil groups. For a section of an elliptic surface to be rational over $\mathbf{Q}$ will mean that $x$ and $y$ are both in $\mathbf{Q}(u)$.

As usual, if an elliptic curve or surface is given by the Weierstrass equation $y^{2}=4 x^{3}-G_{2} x-G_{3}$, then $\Delta$ represents $G_{2}^{3}-27 G_{3}^{2}$, and $J=12^{3} G_{2}^{3} / \Delta$.

In $\S 2$, we use multiple sums and products extensively; in that section, we delete the limits of summation, but we include them here, for the sake of clarity:

$$
\begin{gathered}
\sum B_{i}=\sum_{i=1}^{3} B_{i}, \quad \sum B_{i} B_{j}=\sum_{1 \leq i<j \leq 4} B_{i} B_{j} \\
\sum B_{i} B_{j} B_{k}=\sum_{1 \leq i<j<k \leq 4} B_{i} B_{j} B_{k}, \quad \text { and } \quad \prod B_{i}=\prod_{i=1}^{4} B_{i} .
\end{gathered}
$$

In $\S 3$, we talk about distinct sections of an elliptic surface $E \rightarrow B$, by which we will mean distinct up to sign; that is, if $\sigma=(x, y)$ and $\tau=(x,-y)$, then $\sigma$ and $\tau$ are inverses in the Mordell-Weil group, and will not be thought of as being "distinct." So for two sections to be distinct will mean that they have distinct $x$-coordinates.

2. Parametrization of the family of elliptic surfaces. Suppose we are given an elliptic surface $E \rightarrow B=\mathbf{P}_{1}(\mathbf{C})$, with the surface $E$ having geometric genus $p_{g}$ equal to zero. Work of Kas [4] states that the surface has a Weierstrass equation

$$
y^{2}=4\left[x^{3}-\sum_{i=0}^{4} a_{i} u^{i} x+\sum_{j=0}^{6} b_{j} u^{j}\right]
$$

relative to a suitable parameter, $u$, for the base. Furthermore, suppose the Néron minimal model for $E \rightarrow B$ has a fiber of Kodaira type $\mathrm{I}_{0}^{*}$. We may assume that 
this fiber is over $u=\infty$. (If $J \notin \mathbf{C}$, there can only be one fiber of type $\mathrm{I}_{0}^{*}$ because $p_{g}=0$.) Then the surface has a Weierstrass equation

$$
y^{2}=4\left[x^{3}-\left(a_{2} u^{2}+a_{1} u+a_{0}\right) x+b_{3} u^{3}+b_{2} u^{2}+b_{1} u+b_{0}\right]
$$

Let $E_{(a, b)} \rightarrow B=\mathbf{P}_{1}(\mathbf{C})$ be the elliptic surface given by $(2.1)$, with $(a, b)=$ $\left(a_{2}, a_{1}, a_{0} ; b_{3}, b_{2}, b_{1}, b_{0}\right) \in \mathbf{C}^{3} \times \mathbf{C}^{4}$.

Let $W=\left\{(a, b) \mid\right.$ the Néron model of the elliptic surface $E_{(a, b)} \rightarrow B$ has a fiber of type $\mathrm{I}_{0}^{*}$ above $u=\infty$; and $J \notin \mathbf{C}$ \}. We now give a parametrization of $W$, such that the sections of $E_{(a, b)} \rightarrow B$ are rational in terms of the parameters. Let the parameter space be $W_{1} \cup W_{2}$, where

$$
\begin{array}{r}
W_{1}=\left\{\left(a_{1}, A, D, C_{1}, C_{2}, C_{3}, C_{4}\right) \mid D\left(D^{2}-9 A^{2}\right)\left(3 A^{2}+D^{2}\right) \neq 0,\right. \\
\left.\left(C_{1}, C_{2}, C_{3}, C_{4}\right) \neq(0,0,0,0)\right\}
\end{array}
$$

and

$$
\begin{array}{r}
W_{2}=\left\{\left(b_{2}, A, D, C_{1}, C_{2}, C_{3}, C_{4}\right) \mid A D\left(D^{2}-9 A^{2}\right)\left(A^{2}-D^{2}\right) \neq 0,\right. \\
\left.\left(C_{1}, C_{2}, C_{3}, C_{4}\right) \neq(0,0,0,0)\right\} .
\end{array}
$$

Then define

$$
\begin{aligned}
a_{2} & =\left(3 A^{2}+D^{2}\right) / 4 \\
b_{3} & =A\left(D^{2}-A^{2}\right) / 4 \\
b_{2} & =\left(D^{3} \sum C_{i}^{2}+18 a_{1} b_{3}\right) /\left(12 a_{2}\right) \quad \text { on } W_{1}, \\
& \text { or } \\
a_{1} & =\left(12 a_{2} b_{2}-D^{2} \sum C_{i}^{2}\right) /\left(18 b_{3}\right) \quad \text { on } W_{2}, \\
B_{i} & =4\left(C_{i}^{2}+a_{1} A-b_{2}\right) /\left(9 A^{2}-D^{2}\right), \quad i=1,2,3,4, \\
\left(a_{0} A-b_{1}\right) & =\frac{16\left(3 A\left(a_{1} A-b_{2}\right)^{2}-a_{1}\left(a_{1} A-b_{2}\right)\left(3 A^{2}-a_{2}\right)-D \prod C_{i}\right)}{\left(9 A^{2}-D^{2}\right)^{2}}, \\
a_{0} & =\frac{D^{2} \sum B_{i} B_{j}+6 A\left(a_{0} A-b_{1}\right)-a_{1}^{2}}{\left(9 A^{2}-D^{2}\right)} \\
b_{1} & =a_{0} A-\left(a_{0} A-b_{1}\right), \\
b_{0} & =\frac{2 a_{1}\left(a_{0} A-b_{1}\right)-4 a_{0}\left(a_{1} A-b_{2}\right)+D^{2} \sum B_{i} B_{j} B_{k}}{\left(9 A^{2}-D^{2}\right)} .
\end{aligned}
$$

THEOREM 2.1. The Néron model of the elliptic surface $E_{(a, b)} \rightarrow B$ has a fiber of type $\mathrm{I}_{0}^{*}$ above $u=\infty$, and has nonconstant $J$-invariant if and only if $(a, b)$ is given by the above parametrization.

Proof. Suppose $x=A u+B$. In order for

$$
(A u+B)^{3}-\left(a_{2} u^{2}+a_{1} u+a_{0}\right)(A u+B)+b_{3} u^{3}+b_{2} u^{2}+b_{1} u+b_{0}
$$

to be a square in $\mathbf{C}(u)$, two conditions must hold:

$$
A^{3}-a_{2} A+b_{3}=0
$$

and

(2.3) $\left(3 A B^{2}-a_{0} A-a_{1} B+b_{1}\right)^{2}-4\left(3 A^{2} B-a_{1} A-a_{2} B+b_{2}\right)\left(B^{3}-a_{0} B+b_{0}\right)=0$. 
Let the Weierstrass equation (2.1) be given. We will show (in Lemma 3.4, below) that if $(a, b) \in W$, then equation (2.2) has three distinct solutions for $A$ (over $\mathbf{C}$ ) and, for each $A$, equation (2.3) has four (not necessarily distinct) solutions for $B$ (over $\mathbf{C}$ ). Let $A$ be one of the solutions of (2.2), and let $B_{1}, B_{2}, B_{3}$, and $B_{4}$ be the solutions of (2.3). Let $C_{i}$ be fixed such that

$$
C_{i}^{2}=\left(3 A^{2}-a_{2}\right) B_{i}-\left(a_{1} A-b_{2}\right) ;
$$

let the left side of equation (2.3) be factored as $K \Pi\left(B-B_{i}\right)$; and let $D$ be a fixed square root of $K$.

Then we equate the coefficients of the powers of $B$ in the polynomial equation

$$
\begin{aligned}
& \left(3 A B^{2}-a_{0} A-a_{1} B+b_{1}\right)^{2} \\
& \quad-4\left(3 A^{2} B-a_{1} A-a_{2} B+b_{2}\right)\left(B^{3}-a_{0} B+b_{0}\right)=K \prod\left(B-B_{i}\right),
\end{aligned}
$$

and obtain the equations

$$
\begin{gathered}
9 A^{2}-4\left(3 A^{2}-a_{2}\right)=K, \\
-6 a_{1} A+4\left(a_{1} A-b_{2}\right)=-K \sum B_{i}, \\
a_{1}^{2}-6\left(a_{0} A-b_{1}\right) A+4 a_{0}\left(3 A^{2}-a_{2}\right)=K \sum B_{i} B_{j}, \\
2 a_{1}\left(a_{0} A-b_{1}\right)-4\left(3 A^{2}-a_{2}\right) b_{0}-4 a_{0}\left(a_{1} A-b_{2}\right)=-K \sum B_{i} B_{j} B_{k}
\end{gathered}
$$

from the degree $4,3,2$, and 1 terms, respectively, of equation (2.5). In place of the equation obtained by equating the degree 0 terms of equation (2.5), we use the equation obtained by substituting $B_{i}=\left(C_{i}^{2}+a_{1} A-b_{2}\right) /\left(3 A^{2}-a_{2}\right)$ into equation (2.5), then evaluating the left and right sides at $B=\left(a_{1} A-b_{2}\right) /\left(3 A^{2}-a_{2}\right)$, and taking square roots:

$$
3 A\left(\frac{a_{1}-A-b_{2}}{3 A^{2}-a_{2}}\right)^{2}-a_{1}\left(\frac{a_{1} A-b_{2}}{3 A^{2}-a_{2}}\right)-\left(a_{0} A-b_{1}\right)=\frac{D \prod C_{i}}{\left(3 A^{2}-a_{2}\right)^{2}} .
$$

Then the parametrization is obtained by solving equations (2.4) and (2.6)-(2.10).

If the fiber type above $u=\infty$ is not $\mathrm{I}_{0}^{*}$, then $4 a_{2}^{3}-27 b_{3}^{2}=0$. The assumption that the coefficients $(a, b)$ are given by the above parametrization implies that $D\left(9 A^{2}-D^{2}\right)=0$, a contradiction. On the other hand, if the fiber type above $u=\infty$ is $\mathrm{I}_{0}^{*}$, then the coefficients are given by the parametrization, by construction. Since it can be shown that the $J$-invariant of the elliptic surface (given by this parametrization) is constant if and only if $C_{1}=C_{2}=C_{3}=C_{4}=0$, we conclude that $(a, b) \in W$ if and only if $(a, b)$ is given by the above parametrization. Q.E.D.

Note. If $(x, y)$ is a rational solution of $(2.1)$ with $x=A u+B_{i}$, then it can be shown that

$$
y= \pm\left(2 C_{i} u+\frac{48 A\left(C_{i}^{3}+2 C_{i}\left(a_{1} A-b_{2}\right)\right)}{\left(9 A^{2}-D^{2}\right)^{2}}-\frac{4 a_{1} C_{i}}{9 A^{2}-D^{2}}+\frac{16 D \prod_{j \neq i} C_{j}}{\left(9 A^{2}-D^{2}\right)^{2}}\right) .
$$

Thus, we get the following

COROLLARY 2.2. If the parameters are rational numbers, then the coefficients of equation (2.1) are rational, and the sections with $x=A u+B_{i}$ are rational over Q.

Generators of the Mordell-Weil groups are described in Theorem 3.2, below. 
3. Generators of the Mordell-Weil group. Again, consider the family of elliptic fibrations $E_{(a, b)} \rightarrow B=\mathbf{P}_{1}(\mathbf{C})\left((a, b) \in \mathbf{C}^{3} \times \mathbf{C}^{4}\right)$, given by

$$
y^{2}=4\left[x^{3}-\left(a_{2} u^{2}+a_{1} u+a_{0}\right) x+b_{3} u^{3}+b_{2} u^{2}+b_{1} u+b_{0}\right]
$$

again, restrict consideration to the case where $J \notin \mathbf{C}$.

Proposition 3.1. (i) For almost all $(a, b)$, the rank of the Mordell-Weil group of $E_{(a, b)} \rightarrow B$ is 4 .

(ii) For almost all $(a, b)$, one can find solutions $(x, y)$ of equation (3.1) as follows: Let $x=A u+B$, so that

$$
A^{3}-a_{2} A+b_{3}=0
$$

and

(3.3) $\left(3 A B^{2}-a_{1} A-a_{0} B+b_{1}\right)^{2}-4\left(3 A^{2} B-a_{2} B-a_{1} A+b_{2}\right)\left(B^{3}-a_{0} B+b_{0}\right)=0$,

where $y$ is determined up to sign by $x$. Furthermore, for almost all $(a, b)$, there are 12 distinct $x$ 's given by equations (3.2) and (3.3).

ProOF. The first assertion is a consequence of Shioda's formula; the second statement follows from the proof of Theorem 2.1. Q.E.D.

THEOREM 3.2. If the rank of the Mordell-Weil group of $E_{(a, b)} \rightarrow B$ is 4 , then $a$ basis is given by

$x=A u+B_{j} \quad$ (with one fixed $A$ and 3 distinct $B_{j}$ 's corresponding to $A$ ) and

$$
x=A^{\prime} u+B^{\prime} \quad\left(\text { with } A \neq A^{\prime}, \text { and } B^{\prime} \text { corresponding to } A^{\prime}\right) .
$$

This theorem is proved by a number of lemmas.

LEMMA 3.3. If the rank of the Mordell-Weil group is 4, and there are 12 distinct $x$ 's given by Proposition 3.1, then a basis is as in Theorem 3.2.

PROOF. This is proved in [11].

Recall the definition of the set $W$ from $\S 2$.

LEMMA 3.4. (i) If the Mordell-Weil rank $r$ of $E_{(a, b)} \rightarrow B$ is 4 , then $(a, b) \in W$.

(ii) If $r=4$, then equation (3.2) has three distinct solutions for $A$.

(iii) If $r=4$, then equation (3.3) has, for each $A$ (a solution of (3.2)), degree 4 in $B$.

Proof. (i) is true, because, if the fiber above $u=\infty$ of the Néron model is anything other than $\mathrm{I}_{0}^{*}$, then $r \leq 3$.

(ii) follows, because, if the discriminant $4 a_{2}^{3}-27 b_{3}^{2}$ of the cubic $A^{3}-a_{2} A+b_{3}$ vanishes, then so does the degree 6 term of

$$
\Delta=\left[4\left(a_{2} u^{2}+a_{1} u+a_{0}\right)\right]^{3}-27\left[4\left(b_{3} u^{3}+b_{2} u^{2}+b_{1} u+b_{0}\right)\right]^{2},
$$

and the fiber above $u=\infty$ is not $\mathrm{I}_{0}^{*}$.

(iii). Suppose the coefficient of $B^{4}$ vanishes; then $a_{2}=\frac{3}{4} A_{0}^{2}$ for $A_{0}$ a solution of (3.2). Thus, equation (3.2) is

$$
A^{3}-\frac{3}{4} A_{0}^{2} A+b_{3}=0
$$

Since $A_{0}$ is one of the solutions of $(3.2), b_{3}=-\frac{1}{4} A_{0}^{3}$, and the discriminant $4 a_{2}^{3}-27 b_{3}^{2}$ vanishes. Q.E.D. 
LEMMA 3.5. (i) If, for some $A$ (a solution of (3.2)), there are exactly three corresponding $B$ 's, then $r \leq 3$.

(ii) If, for some $A$ (a solution of (3.2)), there are fewer than three $B$ 's, then $r \leq 3$.

Part (ii) follows from (i), because of Lemma 3.7: that the set $\{(a, b) \mid r \leq 3\}$ is closed; and because the parametrization of $\S 2$ shows that the case in (ii) is a limit point of cases in (i).

PROOF OF (i). Let

$$
\begin{gathered}
W=\left\{(a, b) \mid \text { the Néron model of the elliptic surface } E_{(a, b)} \rightarrow B\right. \text { has } \\
\text { a fiber of type } \left.\mathrm{I}_{0}^{*} \text { above } u=\infty ; \text { and } J \notin \mathbf{C}\right\}, \\
U=\left\{(a, b) \in W \mid \text { the Mordell-Weil rank of } E_{(a, b)} \rightarrow B \text { is } 4\right\},
\end{gathered}
$$

and

$V=\{(a, b) \in U \mid$ there are 12 distinct solutions given by Proposition 3.1 $\}$.

Note that $V$ is a Zariski open subset of $U$, that $U$ is a Zariski open subset of $W$, and that $W$ is a Zariski open subset of $\mathbf{C}^{7}$.

Let $(h, k)$ be a point of $W$ for which there are, for one $A_{i}$ (say $A_{1}$ ), exactly three $B_{i j}$ 's, one of which is a double root of equation (3.3); say $B_{11}=B_{12}, B_{13}$, and $B_{14}$ are the $B_{i j}$ 's, with $B_{12}, B_{13}$, and $B_{14}$ distinct. Furthermore, by Lemma 3.4, we may assume that there is another solution with $x=A_{2} u+B_{21}$ (or else $r \leq 3$ ).

One can view the $A_{i}$ 's and $B_{i j}$ 's as being continuous multivalued functions of $(a, b)$, such that $B_{11}$ and $B_{12}$ coincide when $(a, b)=(h, k)$.

Let $\sigma_{1}, \sigma_{2}, \sigma_{3}, \sigma_{4}$, and $\sigma_{5}$ be the sections defined, up to sign, by

$$
\begin{gathered}
x_{1}=A_{1} u+B_{11}, \quad x_{2}=A_{1} u+B_{12}, \quad x_{3}=A_{1} u+B_{13}, \\
x_{4}=A_{1} u+B_{14}, \quad \text { and } x_{5}=A_{2} u+B_{21} .
\end{gathered}
$$
Then

For $(a, b) \in V,\left\{\sigma_{2}, \sigma_{3}, \sigma_{4}, \sigma_{5}\right\}$ is a basis of the Mordell-Weil group of $E_{(a, b)} \rightarrow B$.

$$
\sigma_{1}=\sum_{i=2}^{5} \alpha_{i} \sigma_{i},
$$

where the $\alpha_{i}$ 's are continuous, integer valued functions on $V$, hence constant on connected components. But $V$ is a Zariski open subset of $\mathbf{C}^{7}$, and hence is connected; so the $\alpha_{i}$ 's are constant on $V$, and relation (3.4) holds on the closure of $V$ in $U$, i.e. on $U$ itself.

Suppose $(h, k) \in U$, that is, suppose the Mordell-Weil rank of the fibration $E_{(h, k)} \rightarrow B$ is 4 . Then $\left\{\sigma_{2}, \sigma_{3}, \sigma_{4}, \sigma_{5}\right\}$ is a basis. But $\pm \sigma_{2}=\sigma_{1}=\sum_{i=2}^{5} \alpha_{i} \sigma_{i}$ shows that $\alpha_{2}= \pm 1$ and $\alpha_{3}=\alpha_{4}=\alpha_{5}=0$; and this contradicts the fact that $B_{11}$ and $B_{12}$ are distinct on $V$. Q.E.D.

LEMMA 3.6. Let $S$ be a topological space, and let $A \subset S$ be open. If $B \subset S-A$ is open (in the relative topology), then $A \cup B$ is open in $S$.

Proof. Since $S-A-B$ is a relatively closed subset of $S-A$, it is the intersection of a closed subset of $S$ with $S-A$, i.e., the intersection of two closed subsets of $S$. Thus $A \cup B$ is open. Q.E.D. 
Recall the definitions

$W=\left\{(a, b) \notin \mathbf{C}^{7} \mid J \notin \mathbf{C}\right.$, and $E_{(a, b)} \rightarrow B$ has a singular fiber of type $\mathrm{I}_{0}^{*}$ over $\left.\infty\right\}$ and

$$
U=\left\{(a, b) \in W \mid E_{(a, b)} \rightarrow B \text { has Mordell-Weil rank 4 }\right\} .
$$

LEMMA 3.7. $U$ is Zariski open in $\mathbf{C}^{7}$.

PROOF. Since we assume that $\Delta(u)=4 a(u)^{3}-27 b(u)^{2}=\delta_{6} u^{6}+\cdots+\delta_{0} u^{0}$ is a sixth degree polynomial (in $u$ ) for $(a, b) \in W$, the discriminant of $\Delta$, disc $(\Delta)$ ), is the resultant of $\Delta(u)$ and $\Delta^{\prime}(u)$, i.e., the determinant of the $11 \times 11$ matrix

$$
\operatorname{disc}(\Delta)=\left|\begin{array}{ccccccccc}
\delta_{6} & \delta_{5} & \cdot & \cdot & \cdot & \delta_{0} & & \\
& \cdot & & & & & \cdot & \\
& & \cdot & & & & & \cdot \\
6 \delta_{6} & 5 \delta_{5} & \cdot & \cdot & \delta_{1} & & & & \\
& \cdot & & & & \cdot & & \\
& & \cdot & & & & \cdot & \\
& & & \cdot & & & & & \cdot \\
& & & & 6 \delta_{6} & 5 \delta_{5} & \cdot & \cdot & \delta_{1}
\end{array}\right|
$$

where the $\delta_{k}$ 's are functions of the $a_{i}$ 's and the $b_{j}$ 's. Thus, $\operatorname{disc}(\Delta)$ is a polynomial function of $(a, b)$, which is zero if $\Delta(u)$ has a multiple root.

Similarly, $E(a, b) \rightarrow B$ has a fiber of type II, III, or IV if the resultant

$$
\left.R(a, b)=\mid \begin{array}{lllll}
b_{3} & b_{2} & b_{1} & b_{0} & \\
& b_{3} & b_{2} & b_{1} & b_{0} \\
a_{2} & a_{1} & a_{0} & & \\
& a_{2} & a_{1} & a_{0} & \\
& & a_{2} & a_{1} & a_{0}
\end{array}\right]
$$

is zero. If $a(u)$ and $b(u)$ have a common root, then $\Delta(u)$ has a multiple root; hence $R(a, b)$ must divide $\operatorname{disc}(\Delta)$.

Let $Q(a, b)$ and $R_{1}(a, b)$ be polynomials so that $\operatorname{disc}(\Delta)=Q(a, b) R_{1}(a, b)$, with $(Q(a, b), R(a, b))=1$ and $R_{1}(a, b)=0 \Leftrightarrow R(a, b)=0$. (In one example, I have computed that $R_{1}(a, b)=R(a, b)^{3}$; I suspect that, in general, $R_{1}$ is $R$, raised to some power.) If $R(a, b) \neq 0$ and $\operatorname{disc}(\Delta)=0$, then $Q(a, b)=0$; in other words, $Q(a, b)=0$ if $E_{(a, b)} \rightarrow B$ has a singular fiber of type $\mathrm{I}_{\nu}$, with $\nu>1$.

Let $U_{0}\{(a, b) \in W \mid \operatorname{disc}(\Delta) \neq 0\}$. Then for $(a, b) \in U_{0}, \Delta(u)$ has no repeated roots, and $E_{(a, b)} \rightarrow B$ has an $\mathrm{I}_{0}^{*}$ above $u=\infty$, and six singular fibers of type $\mathrm{I}_{1}$. $U_{0}$ is Zariski open, and is contained in $U$.

Let

$$
\begin{array}{r}
U_{1}=\left\{(a, b) \in W \mid E_{(a, b)} \rightarrow B \text { has four singular fibers of type } \mathrm{I}_{1},\right. \\
\text { and a singular fiber of type II }\}
\end{array}
$$

and let

$$
U_{2}=\left\{(a, b) \in W \mid E_{(a, b)} \rightarrow B \text { has two singular fibers of type } \mathrm{I}_{1},\right.
$$
and two singular fibers of type II $\}$. 
Note that $U=U_{0} \cup U_{1} \cup U_{2}$. We now observe that $U_{1}$ is Zariski open in $W-U_{0}$, and that $U_{2}$ is Zariski open in $W-U_{0}-U_{1}$, which will prove the result, by the previous lemma.

$U_{1}$ may be described by the following conditions.

(1) $J \notin \mathbf{C}, \operatorname{deg}(\Delta)=6$;

(2) $\operatorname{disc}(\Delta)=0$ (so $U_{1} \subset W-U_{0}$ );

(3) $Q(a, b) \neq 0$; and

(4) the greatest common divisor of $a^{2}$ and $b$ is linear.

These can be translated into polynomail conditions on $(a, b)$, and it can be seen that $U_{1}$ is relatively open in $W-U_{0}$.

Similarly, $U_{2}$ may be described by:

(1) $J \notin \mathbf{C}, \operatorname{deg}(\Delta)=6$

(2) $\operatorname{disc}(\Delta)=0$

(3) $Q(a, b) \neq 0$;

(4) $b(u)$ is square free; and

(5) the greatest common divisor of $a$ and $b$ is quadratic.

These can also be translated into polynomial conditions on $(a, b)$, and it can be seen that $U_{2}$ is relatively open in $W-U_{0}-U_{1}$.

Therefore, $U=U_{0} \cup U_{1} \cup U_{2}$ is Zariski open in $W$. Q.E.D.

This concludes the proof of Theorem 3.2. I have been told that Lemma 3.7 is true much more generally: that the rank of the parabolic cohomology is lower semicontinuous; but I do not know the proof.

\section{BIBLIOGRAPHY}

1. A. Brumer and K. Kramer, The rank of elliptic curves, Duke Math. J. 44 (1977), 715-743.

2. D. A. Cox and S. Zucker, Intersection numbers of sections of elliptic surfaces, Invent. Math. 53 (1979), 1-44.

3. F. J. Grunewald and R. Zimmert, Über einige Rationale Elliptische Kurven mit Freien Rang $\geq 8$, J. Reine Angew. Math. 296 (1977), 100-107.

4. A. Kas, Weierstrass normal forms and invariants of elliptic surfaces, Trans. Amer. Math. Soc. 225 (1977), 259-266.

5. Ju. I. Manin, The Tate height of points on an abelian variety. Its variants and applications, Amer. Math. Soc. Transl. (2) 59 (1966), 82-110.

6. K. Nakata, On some elliptic curves defined over $Q$ of free rank $\geq 9$, Manuscripta Math. 29 (1979), 183-194.

7. A. Néron, Problèmes arithmétiques et géométriques, rattachés a la notion de rang d'une courbe algébrique dans un corps, Bull. Soc. Math. France 80 (1952), 101-166.

8. __ Propriétés arithmétiques de certaines familles de courbes algébriques, Proc. Internat. Congress, Amsterdam (1954), vol. III, North-Holland, Amsterdam, 1956, pp. 481-488.

9. D. E. Penney and C. Pomerance, Three elliptic curves with rank at least seven, Math. Comp. 29 (1975), 965-967.

10. I. Safarevið et al., Algebraic surfaces, Proc. Steklov Institute, Moscow, 1965; English transl., Amer. Math. Soc., Providence, R. I., 1967.

11. C. F. Schwartz, A Mordell-Weil group of rank 8, and a subgroup of finite index, Nagoya Math. 93 (1984).

12. __ An elliptic surface with Mordell-Weil rank 8 over the rational numbers (in preparation).

13. T. Shioda, On elliptic modular surfaces, J. Math. Soc. Japan 24 (1972), 20-57.

\footnotetext{
Department of Mathematics, Rider College, Lawrenceville, New Jersey 08648
} 